\title{
A restraint system for the psychophysiological study of dogs ${ }^{1}$
}

D. E. ANDERSON, L. A. DALEY, J. D. FINDLEY, and J. V. BRADY, ${ }^{2}$ Department of Psychiatry and Behavioral Science, THE JOHNS HOPKINS UNIVERSITY SCHOOL OF MEDICINE, Baltimore, Maryland 21205

The present report describes a system for continuous psychophysiological study of the dog in a relatively flexible restraint harness that maintains the animal in a constant directional orientation, but allows him to assume any of a number of standing, sitting, or lying positions. The system permits the concurrent monitoring of behavioral and physiological processes under both acute and chronic conditions. $A$ harness consisting of a leather collar and two body yokes is suspended from a pivoting metal hanger assembly. The harness is integrated with an isolation hamber equipped with automatic food, ater, stimulus, and response devices. Techniques for installing and maintaining dogs in the harness are described. A system for the continuous monitoring of cardiovascular functions and some experimental uses of the restraint system are suggested.

Laboratory studies of psychophysiological processes in the unanesthetized behaving dog continue to require some form of restraint in order to insure reliable monitoring. The classical restraint system was devised by Pavlov (1927) and has been used, with only minor variations, by most investigators (Gantt, 1960). Similar restraint systems for the dog have been described more recently by Kaplan, Campbell, Martin, Wulp, and Lipinski (1962) and Reininger (1969). With all of these devices, however, severe limitations are imposed upon the length of the restraint period by the relatively rigid positioning of the animal, and continuous monitoring of physiological processes during experimental intervals longer than a few hours has seldom been possible.

The present report describes a system for continuous long-term psychophysiological study of the dog in a relatively flexible restraint harness that permits the animal to assume any of a number of standing, sitting, or lying

Fig. 1. Illustration of restraint apparatus in use. positions. The animal is maintained in a constant directional orientation by only two loose yokes at the shoulders and waist that prevent escape and restrict access to chronically indwelling catheters and associated transducers. In addition, the restraining harness is integrated into a relatively sound- and light-resistant isolation chamber equipped with automatic feeding, watering, and stimulus and response devices. The experimental system thus provides for the long-term investigation of behavioral-physiological interactions and metabolic changes in conscious laboratory dogs under relatively comfortable conditions. Although animals of various weights and sizes have been restrained in the harness, the dimensions, construction details, and applications described in this report have been most extensively tested with standard medium-sized beagles in the 20- to $25-1 \mathrm{~b}$ weight range.

\section{GENERAL CONSTRUCTION}

The gross features of the apparatus in use are shown in Fig. 1. The system is enclosed by two Formica-lined cabinets, constructed one within the other, as illustrated diagramatically in Figs. 2 and 3.
The component parts are labeled and/or lettered to clarify the description in the text. A removable panel door with built-in viewing window encloses the inner chamber and permits ready access to the dog. The overall height of the outer chamber is approximately 44 in. and the base measures $30 \times 45 \mathrm{in}$. The harness assembly is counterbalanced and suspended from a slotted brass center support, A, which attaches ridigly to the inside top of the outer cabinet and the outside top of the inner chamber as shown in Figs. 1, 2, and 3. An adjustable aluminum crossbar, B (16 in.), and pivoting hanger assembly, $C$ (18 in.), attach at the center of the upper crossbar, B, to the slotted support, A, with a $1 / 4$-in. shoulder bolt upon which the entire assembly pivots and moves vertically. The hangers, $\mathrm{C}$, and lower crossbar, D (12 in.), project into the inner chamber through a longitudinal slot which permits free fore and aft movement (Fig. 2) but restricts lateral displacement. Nylon ropes (approximately $10 \mathrm{ft}$ of $1 / 4$-in.), block pulleys ( $3 / 8 \mathrm{in.}$ W $\times 1 \frac{11 / 4}{\mathrm{in}}$. diam), and lead counterweights, E (3-1b front, 2-1b rear), suspended in the space between the outer and inner chambers as shown in Figs. 2 and 3 , provide a counterbalance system that

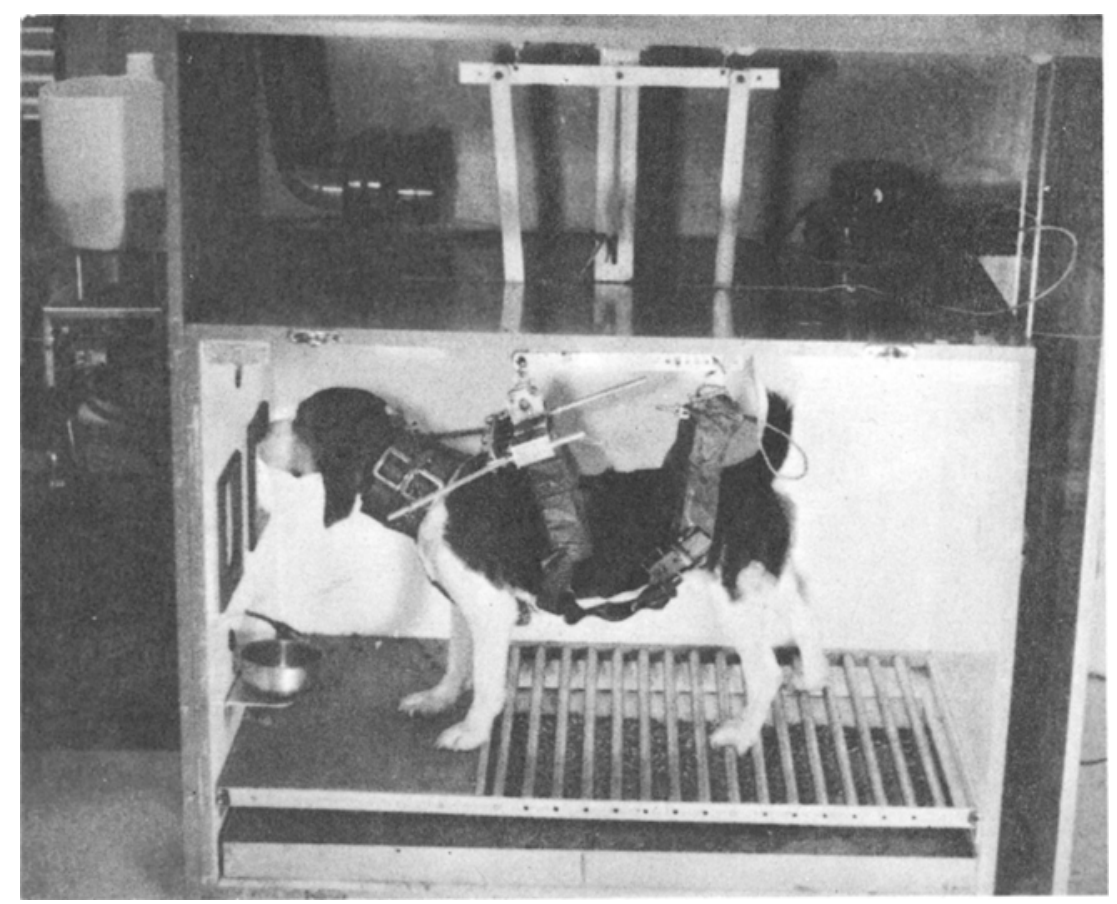




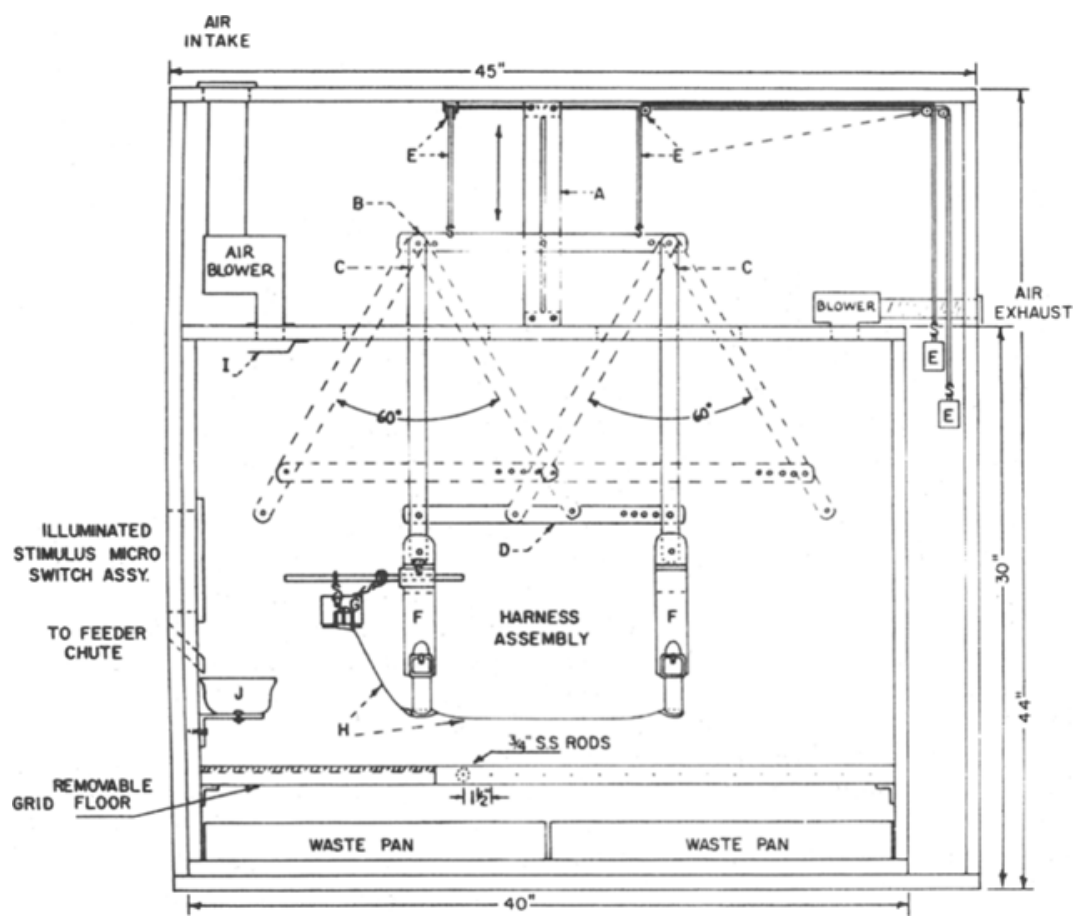

permits position change by the animal with relative ease.

The two yokes, F, that hold the dog, fasten to the free-moving ends of the fore and aft hangers, $C$, as shown in Figs. 4 and 5. The upper portion of each yoke is constructed of 2 -in. clear white pine with a 1 -in. layer of polyurethane foam cemented to the inside curvature as indicated in Fig. 6. The entire yoke is covered with denim, and a soft broad leather belt and buckle are fastened to the underside of the

yoke as shown in Figs. 4, 5, and 6, to provide a loose adjustable fitting around the animal's body. A collar support constructed of two 3/8-in. aluminum rods, formed as shown in Fig. 7, is mounted on top of the front yoke as indicated (Figs. 4, 5 , and 6). Slip rings and light adjustable-length chains on either side of the dog's head attach the support to a soft, wide, custom-fitted leather collar, $G$, in Fig. 2 (9-02 hide, 6 in. wide) around the animal's neck (Figs. 1 and 5 ). The collar is,

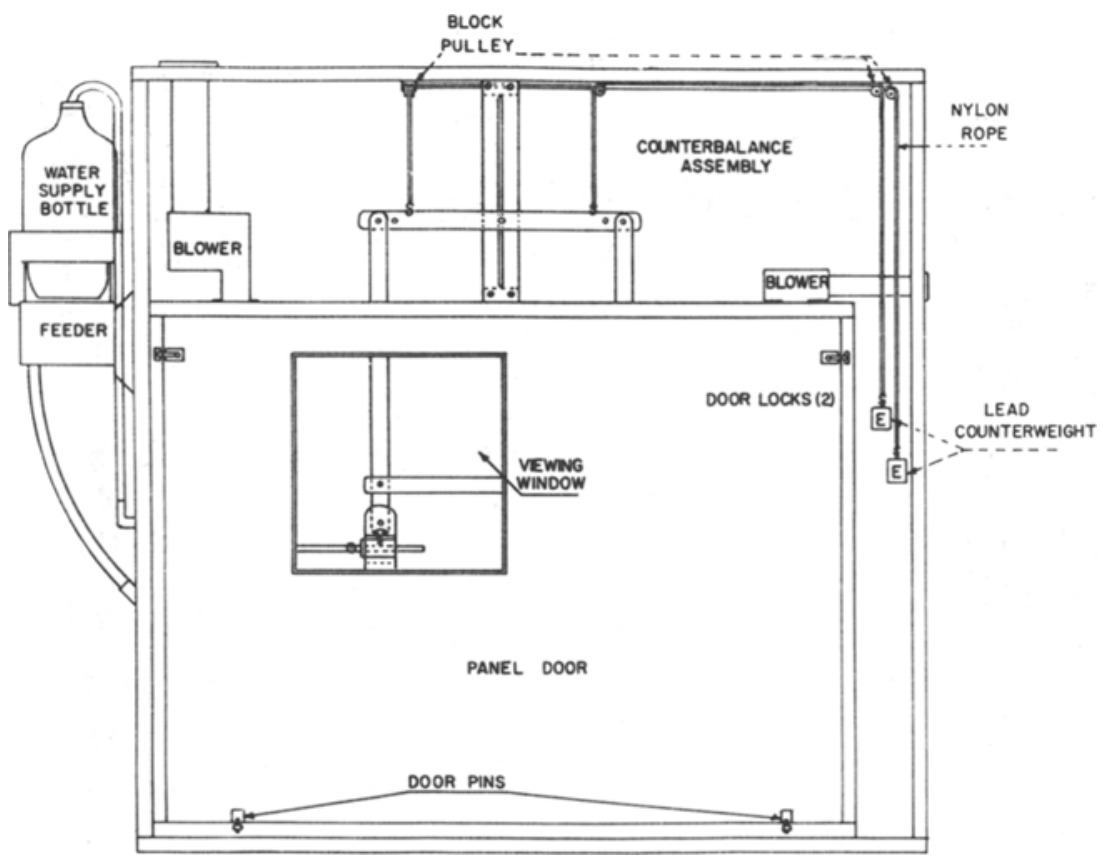

Fig. 2. Diagram showing internal construction of restraint system and surrounding environment. The designation of components by letter is to furnish a basis of reference for description of apparatus in text.
Legend.
A. Slotted brass $c$ enter support
B. A luminum crossbar (upper)
C. Pivoting hanger assembly
D. Aluminum crossbar (lower)
E. Nylon rope, block pulleys, lead coun- terweights
F. Yokes
G. Leather collar
H. Leather "stringer"
1. Intake deflector plate
J. Feed bowl

in turn, connected to the front and rear yokes by an additional leather "stringer," $\mathrm{H}$ in Fig. 2, which runs along the ventral surface of the body (Figs. 1, 2, and 4). This arrangement limits extreme head movements that might damage indwelling catheters and associated transducers without requiring undue restraint.

The animal's weight is supported by a grid floor constructed of $3 / 4$-in. stainless steel tubing recessed into an aluminum frame on $1 \frac{1}{2}$-in. centers (Figs. 1 and 2). A fiberboard strip covers the forward section of the grip to provide more stable footing under the dog's front paws (Fig. 1), and two removable galvanized steel (22-ga) waste pans $(24 \times 24$ in.) lined with Pel-E-Cel (disposable absorbent waste material) are recessed under the grid floor (Fig. 2).

Ventilation and temperature control of the inner chamber is accomplished by means of a forced-air blower and exhaust system mounted in the top front and top rear of the enclosure, as shown in Figs. 1, 2, and 3. An intake deflector plate, I (Fig. 2), enhances uniform air dispersal throughout the compartment. Hard wire leads and fine polyethylene catheter connections to the animal can be fed into the chamber either through existing slotted openings or additional small access holes around door panel insulation corners.

\section{FOOD, WATER, AND BEHAVIOR PROGRAMMING}

Figures 1 through 5 show the front panel of the inner chamber facing the dog, equipped with an easily accessible stainless steel bowl-type food tray, J, fixed in place below a feeder chute that dispenses dry dog food pellets (Ken-L-Ration, Gaines, Wayne's) from a remotely controlled and

Fig. 3. Diagram showing external view of compartment. 


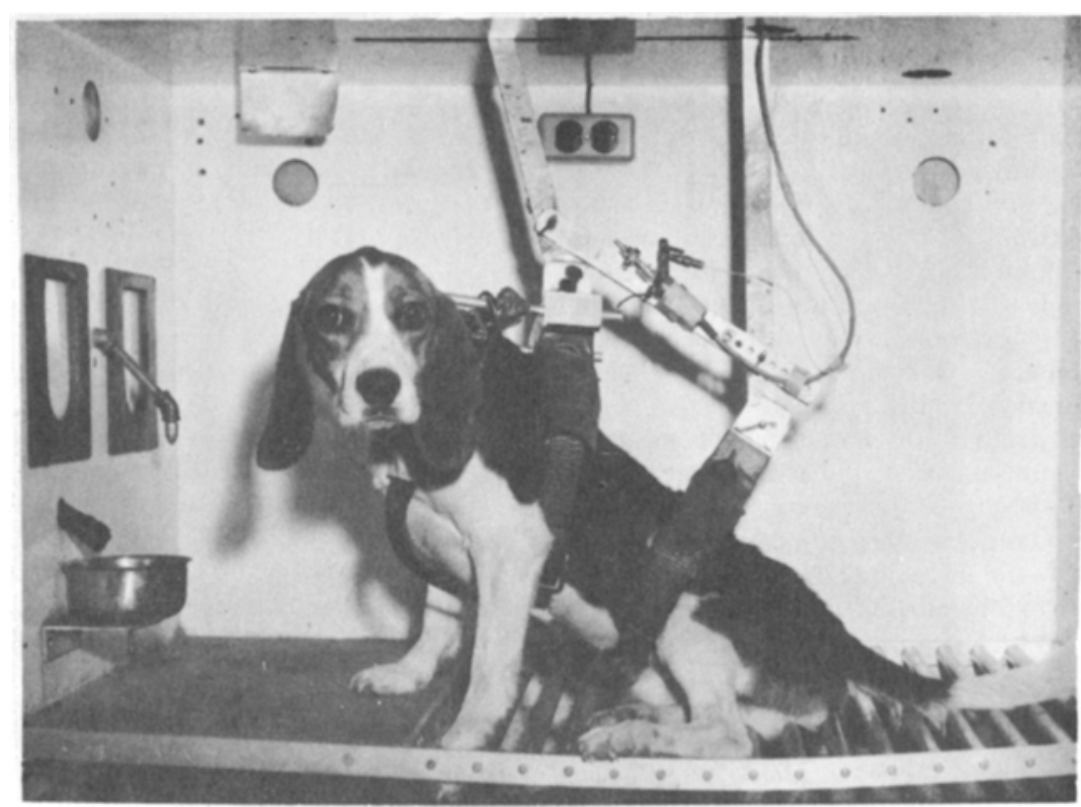

programmable automatic feeder (BRS-Foringer). Ad lib access to water is provided by a Hart poultry valve mounted above the food tray (Fig. 4) and supplied by a gravity-feed tubing system from a 1-gal refillable plastic reservoir. Both the automatic feeder and water reservoir are mounted on the outside wall of the chamber for ready access (Figs. 1 and 3). Two large ( $1 / 4 \times 3 \times 5$ in.) translucent white Plexiglas response keys, each transilluminated from behind with two programmable 28-V lamps, are mounted above the food tray and water valve, as
Fig. 4. Dog sitting in restraint harness.

surgical implantation of a polyvinyl catheter (18-ga) into either the femoral or carotid artery. The catheter is tunneled beneath the skin from the point of insertion to the center of the dog's back, where it is exteriorized and connected to a stop-cock valve attached to a Statham straingage transducer (P-23) secured to the lower crossbar. A second stop-cock valve and polyethylene tube connects the transducer to a Harvard peristaltic pump which provides a continuous saline-heparin infusion at a constant rate of approximately $6 \mathrm{ml} / \mathrm{h}$. The polyethylene tube and transducer cable are led out of the inner chamber through the rear yoke hanger slot in the top of the box and thence through a small opening in the outer chamber. The infusion catheter and transducer cable are passed through a conduit to the infusion pump and electronic monitoring system, respectively, in an adjoining room.

The cardiovascular monitoring system consists basically of an electronic averager that provides on-line heart-rate and blood-pressure measurements over selected periods from $10 \mathrm{sec}$ to several hours, as required. The data is recorded in printout form as average heart-rate values in bpm and average systolic and average diastolic blood pressure in $\mathrm{mm} / \mathrm{Hg}$. The transducer output signal is displayed on a cathode-ray oscilloscope that provides both a continuous visual indicator of the blood pressure wave form and a signal for calibration of the transducer. A beat-by-beat digital readout is also provided by large panel-mounted microvolt meters. No polygraph recorder is required.

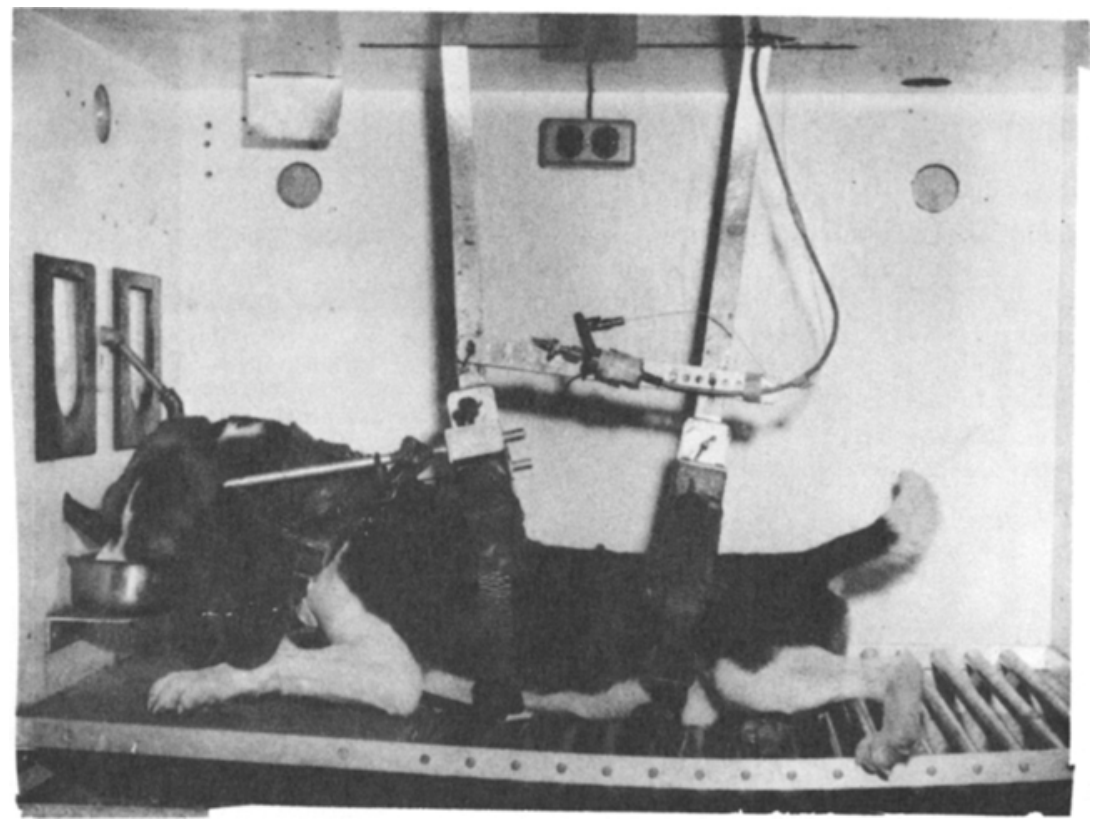

\section{INSTALLATION OF ANIMALS} AND SOURCES OF DIFFICULTY

Several approaches may be used for restraining and positioning animals in the harness, but the following procedure can be effectively followed by one person, though two might be preferable initially. The animal is placed in the standing position on the grid floor facing the front work panel of the inner chamber. The wide leather collar, G, suspended from the slip-ring and aluminum collar support assembly is first adjusted and then fastened around the dog's neck to fit snugly, but not too tightly. Next, the front and rear yokes, F, are adjusted over the dog's back and secured with the attached leather belts and buckles around the animal's body. The front yoke is positioned just behind the

Fig. 5. Dog lying in restraint harness, eating from food tray. 


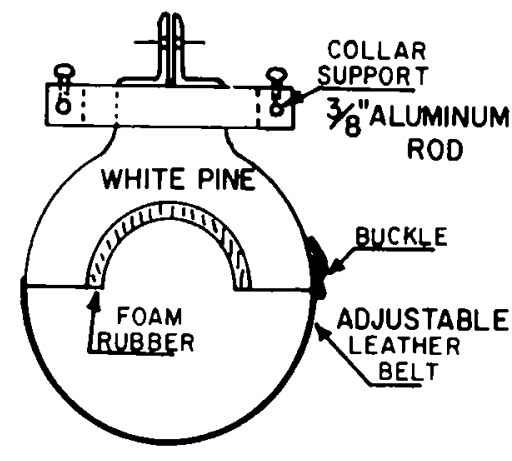

YOKE ASSEMBLY - FRONT VIEW

Fig. 6. Yoke assembly-front view.

fore legs and the rear yoke is positioned just in front of the hind legs (Fig. 1). The leather "stringer," $H$, which runs along the under surface of the dog's body, is then connected to the collar and the two yokes. Necessary adjustments in the "spread" between the two yokes, F, are made by means of the alternate fastening holes on the lower crossbar, D, which fixes and maintains the distance between front and rear yokes, $F$.

If the original positioning of the dog in the harness is carefully checked in several different standing, sitting, and lying positions to insure freedom of movement without compromise of the harness restraint, few difficulties are encountered. The wide range of movement permitted by the harness, however, imposes continuing stresses and strains upon both the hardware and software, which should be checked intermittently during the course of the restraint period. The duration of the restraint period may vary depending upon experimental requirements, but, in general, intervals of up to 4 months have thus far produced no untoward effects. Since critical adjustments are tailored for each dog individually to assure comfortable positioning and to eliminate pressure points, the limits of movements, particularly of the head, must be carefully determined to prevent access to restraining belts and indwelling catheter systems.

\section{EXPERIMENTAL USES}

Restraining devices similar to this harness system have been used with dogs in the past for relatively acute observations during which it was necessary to prevent an animal from reaching lead wires attached to surface electrodes or fistula access sites. In the course of developing a system suitable for continuous cardiovascular monitoring in which both blood-pressure and heart-rate recordings were required over extended time periods, additional applications of the technique have become apparent. For example, the catherized preparation offers unique advantages in pharmacological studies in which frequent injection or continuous infusion are required while concurrent physiological or behavioral changes are measured in the unanesthetized dog. With appropriate accessories, a wide range of biochemical, electrophysiological, and other physiological indices of gastrointestinal activity, respiration, and body temperature can also be measured. The physiological versatility combined with the behavioral assessment features makes the system particularly useful for multidisciplinary studies involving concurrent determinations of interacting biochemical, neurological, physiological, and psychological changes.

An important consideration in evaluating the usefulness of this system is, of course, the extent to which stressful effects are imposed upon the animal by the harness restraint. per sc. There are, of course, marked differences between the degree of restraint involved in this dog harness and the immobilization conventionally used to produce stress in laboratory animals. The positions available approximate those normally assumed by the dog and there is considerable freedom of the extremities. Sleep patterns do not appear to be disturbed and normal food and water intake is maintained. In addition, extensive observations of cardiovascular function have now been made under both resting conditions and during behavioral performances in the harness over continuous restraint periods of several months' duration. Although substantial and consistent elevations in blood pressure and heart rate have been observed to occur in response to specific task requirements involving particularly shock avoidance, resting baselines are

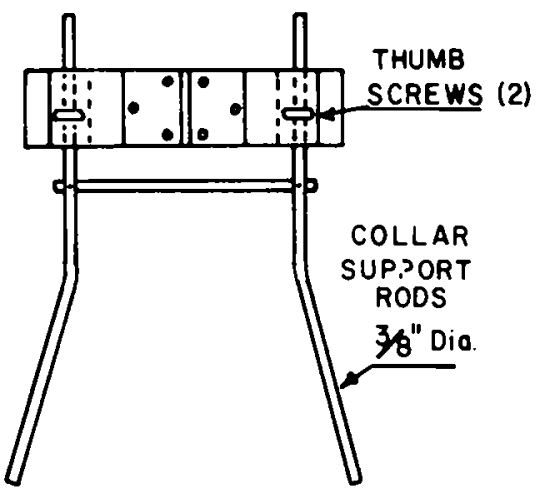

TOP VIEW

Fig. 7. Collar support assembly-top view.

rapidly recovered upon termination of the performance requirements and stable autonomic levels within normal limits are generally maintained. Although there are, of course, limitations to the technique produced, for example, by the restraint and social isolation aspects. the system would seem to have many unique advantages over methods now available for experimental studies involving dogs.

\section{REFERENCES}

GANTT, W. H. Cardiovascular component of the conditional reflex to pain, food, and other stimuli. Physiological Reviews, 1960, 40, 266-291.

KAPLAN, M., CAMPBELL, S. L., MARTIN, J. M., WULP, D. G., \& LIPINSKI, C. E. A restraining device for psychophysiological experimentation with dogs. Joumal of the Experimental Analysis of Behavior, 1962, 5, 209-211.

PAVLOV, I. P. (Translated by G. V. Anrep) Lectures on conditioned reflexes, New York: Dover Publications, 1927. Pp. 16-32.

REININGER, E. J. A body sling to keep dogs in the standing position. Joumal of the Fxperimental Analysis of Behavior, 1969, 12. 462.

\section{NOTES}

1. This project was supported by NIH Grant HE 06945-09.

2. The authors wish to extend their appreciation to several people who participated in the development of this sytem, including Emest Carrington, W. Don Galloway, David Siscovick, Patricia Thiess, and Tracy Wendel. 